\title{
HMGN5 promotes proliferation and invasion via the activation of Wnt/ $\beta$-catenin signaling pathway in pancreatic ductal adenocarcinoma
}

\author{
JIANWEN ZHAO $^{1}$, YONG WANG ${ }^{2}$ and XINGLONG WU ${ }^{3}$ \\ ${ }^{1}$ Scientific Research Center of LanLing County Hospital; ${ }^{2}$ Oncology Department of LanLing County Hospital; \\ ${ }^{3}$ Laboratory of Scientific Research Center of LanLing County Hospital, Linyi, Shandong 277700, P.R. China
}

Received December 5, 2017; Accepted June 29, 2018

DOI: $10.3892 / \mathrm{ol} .2018 .9090$

\begin{abstract}
Pancreatic ductal adenocarcinoma (PDAC) is a highly aggressive carcinoma with a poor prognosis. A recent study has demonstrated that high mobility group nucleosome binding domain 5 (HMGN5) was involved in tumorigenesis and progression of multiple types of human cancers. However, the role of HMGN5 in PDAC is unknown. The objective of the present study was to analyze the function and novel mechanism of HMGN5 involved in PDAC cell progression. It was firstly revealed that the expression of HMGN5 was significantly upregulated in PDAC cell lines and tissues, when compared with that in normal pancreatic duct epithelial cells and adjacent normal pancreatic tissues. In vitro assay revealed that HMGN5 silencing impaired PDAC cell viability, proliferation, migration and invasion. Similarly, tumor growth rate was also decreased in vivo following HMGN5 silencing. Furthermore, it was demonstrated that HMGN5 silencing significantly inhibited epithelial-mesenchymal transition in vitro. Notably, HMGN5medicated Wnt/ $\beta$-catenin signaling pathway activation was observed to be one of the critical signal transduction pathways that associates HMGN5 with EMT activation. Collectively, the results indicated the important role of HMGN5 in PDAC cell proliferation and metastasis, and provide a promising target against the transcriptional program of PDAC.
\end{abstract}

\section{Introduction}

Pancreatic ductal adenocarcinoma (PDAC) is ahead of breast cancer as the third leading cause of cancer-related deaths in the US (1); moreover, PDAC is predicted to become the second

Correspondence to: Professor Xinglong Wu, Laboratory of Scientific Research Center of LanLing County Hospital, 4 JianKang Street, LanLing, LinYi, Shandong 277700, P.R. China

E-mail:wxlong1999@163.com

Key words: high mobility group nucleosome binding domain 5, pancreatic ductal adenocarcinoma, proliferation, migration and invasion, Wnt/ $\beta$-catenin signaling pathway leading cause of cancer-related deaths by 2020 (2). No effective screening test for this malignancy exists, and metastatic disease is commonly present at initial diagnosis. Currently, PDAC has a distinctive adverse prognosis with an overall 5 -year survival rate of $<6 \%$ (2). Therefore, novel and effective therapy approaches for PDAC are urgently needed.

Epithelial-mesenchymal transition (EMT) is a physiological process that allows epithelial cells to acquire the motile and invasive characteristics of mesenchymal cells (3). EMT is a well-coordinated process triggered by many signalling pathways during embryonic development. A recent study has demonstrated that EMT is involved in the progression of neoplasia. Cells undergoing EMT progressively lose the expression of components in the epithelial cell junctions, produce a mesenchymal vimentin cytoskeleton and acquire both invasive and chemoresistance properties. Recent studies have also proposed that metastasis is an early event in the natural history of PDAC (4). Therefore, improving the knowledge of the molecular mechanisms of EMT is essential in designing more effective treatments for this deadly disease.

High-mobility group nucleosome-binding domain 5 (HMGN5) (also named nucleosome-binding protein 1) has been suggested as an oncogene in some types of cancers. A recent study has shown that HMGN5 regulates DNA replication, DNA repair, histone modification and gene transcription in combination with chromatin regulators (5). An increasing number of studies have demonstrated that HMGN5 is overexpressed in many types of cancers, including breast cancer, osteosarcoma, prostate cancer, glioma, lung and renal cancer (6-14). HMGN5 promotes tumour progression by enhancing proliferation, inhibiting apoptosis and promoting metastasis. However, the function and molecular mechanism of HMGN5 in PDAC have not been illustrated. Nevertheless, HMGN5 may be a potential target for developing cancer therapies for PDAC.

The aim of the present study was to illustrate the role and molecular mechanism of HMGN5 in PDAC. Firstly, we detected the HMGN5 expression in PDAC cell lines and normal pancreatic ductal cells. Then, we demonstrated that HMGN5 silencing significantly impaired the PDAC cell viability, proliferation and metastasis and EMT in vitro and retarded the tumour growth in vivo. We demonstrated that the 
HMGN5-mediated Wnt/ $\beta$-catenin signalling pathway is one of the critical signal transduction pathways that link HMGN5 to EMT activation.

\section{Materials and methods}

Cell culture. Human PDAC cell lines (Capan-2, PANC-1, SW1990, COLO357 and MIAPaCa-2) and normal human pancreatic duct epithelial (HPDE) cell line were obtained from Shanghai Cell Bank (Shanghai, China). PDAC cell lines were cultured in Dulbecco's modified Eagle's medium (DMEM) supplemented with $10 \%$ fetal bovine serum (FBS). HPDE cells were maintained in keratinocyte serum-free medium (Gibco; Thermo Fisher Scientific, Inc., Waltham, MA, USA) supplemented with bovine pituitary extract and epidermal growth factor. All cells were cultured at $37^{\circ} \mathrm{C}$ in humidified incubator with $5 \% \mathrm{CO}_{2}$.

Tissue samples. The present study was approved by the Medical Ethics Committee of LanLing County Hospital. All patients included in this research were required to provide written informed consent. Fifteen paired PDAC and adjacent normal tissues were obtained from patients who underwent surgical resection in LanLing County Hospital. All tissue samples were snap-frozen in liquid nitrogen immediately after surgery and stored at $-80^{\circ} \mathrm{C}$ until RNA extraction.

Cell transfection. shRNA plasmids for human HMGN5 were designed against the HMGN5 gene and constructed in Phblvu6-puro vectors (Shanghai GeneChem Co., Ltd., Shanghai, China). The shRNA sequences used in the present study were as follows: shHMGN5 5'-ATGAGAAAGGAGAAGATG C-3' and shcontrol: 5'-GAAGAATATCGAAGGAAGA-3'. To generate stable HMGN5 knockdown cells, PANC-1 cells were grown in 6-well plates until they reached $60 \%$ confluency. The medium was replaced with $1 \mathrm{ml}$ of fresh culture medium supplemented with $100 \mu \mathrm{l}$ viral supernatant $\left(1 \times 10^{8} \mathrm{UT} / \mathrm{ml}\right)$ and $8 \mu \mathrm{g} / \mathrm{ml}$ Polybrene for $24 \mathrm{~h}$. The PANC-1 cells were further cultured in medium containing puromycin at $3 \mu \mathrm{g} / \mathrm{ml}$. Individual puromycin-resistant colonies were isolated during drug screening. The open reading frame of $\beta$-catenin was inserted into pcDNA3.1 vector to generate pcDNA3.1/ $\beta$ catenin overexpression vector. Transient transfection was performed with a Lipofectamine 2000 reagent (Invitrogen; Thermo Fisher Scientific, Inc., Waltham, MA, USA) according to the manufacturer's instructions. Knockdown or overexpression efficiency was examined via western blot assay.

Colony formation assay. PANC-1 cells transfected with shHMGN5 or shcontrol and $\beta$-catenin plasmid were seeded at 200 cells/well in 6-well plates. Seven days later, the colonies were stained with crystal violet, photographed, and then scored.

CCK-8 assay. Cell viability was determined via Cell Counting Kit-8 (CCK-8) (Beyotime Institute of Biotechnology, Haimen, China). Briefly, cells (3,000/well) transfected with shHMGN5 or shcontrol and $\beta$-catenin plasmid in $200 \mu \mathrm{l}$ medium were plated into 96-well plates. Zero, 24, 48 and $72 \mathrm{~h}$ later, $10 \mu \mathrm{l}$ of CCK-8 solution was added into each well. After $2 \mathrm{~h}$, the optical density values of each well were measured at $450 \mathrm{~nm}$ using a microplate reader (Thermo Fisher Scientific, Inc., Waltham, MA, USA). CCK- 8 assay were repeated 3 times.

Transwell assay. Cell metastasis was determined using Boyden chamber assay. For the invasion assay, the upper sides of the filters were coated with $50 \mu \mathrm{l}$ Matrigel (BD Biosciences, Bedford, MA, USA). Cells (5x104) with $200 \mu$ l of serum-free medium were seeded in the upper chamber. The lower chamber was filled with medium supplemented with 5\% FBS. Following incubation at $37^{\circ} \mathrm{C}$ with $5 \% \mathrm{CO}_{2}$ for $8 \mathrm{~h}$ (migration) or $12 \mathrm{~h}$ (invasion), cells on the lower filter were fixed with methanol, stained with crystal violet, and then counted under a light microscope.

Western blot analysis. Equal amounts of the protein from lysates of PDAC cells were subjected to $10 \%$ sodium dodecyl sulfate-polyacrylamide gel electrophoresis (SDS-PAGE) and then transferred to polyvinylidene fluoride (PVDF) membranes. The immunoreactive bands were firstly incubated with the primary antibodies, including HMGN5 (1:500), wnt1 (1:500), total or phosphorylated $\beta$-catenin (1:500), (all from Cell Signaling Technology, Inc., Danvers, MA, USA) E-cadherin (1:1,000), N-cadherin (1:1,000), Vimentin $(1: 1,000)$ (all from Santa Cruz Biotechnology, Inc., Santa Cruz, CA, USA), $\beta$-actin (1:2,000; Beyotime Institute of Biotechnology) overnight at $4^{\circ} \mathrm{C}$ and horseradish peroxidase-conjugated goat anti-rabbit antibody (Santa Cruz Biotechnology, Inc.) at room temperature for $2 \mathrm{~h}$. The intensity of protein bands was detected by Image-Pro Plus 6.0 software. $\beta$-actin served as the loading control.

Reverse transcription-quantitative polymerase chain reaction (RT-qPCR). Total RNA from cells was extracted using TRIzol reagent (Invitrogen; Thermo Fisher Scientific, Inc.) and then reverse-transcribed through a reverse transcription kit (Takara Biotechnology Co., Ltd., Dalian, China) following the manufacturer's protocol. RT-qPCR was conducted with All-in-One ${ }^{\mathrm{TM}}$ miRNA RT-qPCR Detection Kit (AOMDQ020; GeneCopoeia, Inc., Rockville, MD, USA) on CFX96 ${ }^{\mathrm{TM}}$ Real-Time PCR Detection System supplied with analytical software (Bio-Rad Laboratories, Inc., Hercules, CA, USA). The primers used for amplification were: HMGN5, forward 5'-CATGGACATGAGCACAATCA-3' and reverse 5'-CCC TTTTCTGTGGCATCTTC-3'; E-cadherin, forward 5'-GTA CTTGTAATGACACATCTC-3' and reverse 5'-TGCCAG TTTCTGCATCTTGC-3'; N-cadherin, forward 5'-ATCAAA GACCCATCCACC-3' and reverse 5'-CCTCCTCACCACCAC TA-3'; vimentin, forward 5'-CTTCCGCGCCTACGCCA-3' and reverse 5'-GCCCAGGCGACCTACTCC-3'; $\beta$-actin, forward 5'-GATCATTGCTCCTCCTGAGC-3' and reverse, 5'-ACTCCTGCTTGCTGATCCAC-3'.

Tumourigenesis assay in nude mice. The protocols for animal experiment study were approved by the Animal Care and Welfare Committee of LanLing County Hospital and conducted in strict accordance with the guidelines of the National Animal Welfare Law of China. Four-week-old male $B A L B / c$ nude mice $(n=12)$ were purchased from the Laboratory Animal Center of JiLin University (JiLin, China) 
and maintained in a SPF environment. Stable PANC-1 shHMGN5 and PANC-1 shcontrol cells $\left(1 \times 10^{6}\right)$ were subcutaneously injected into the right flanks of athymic nude mice. Tumor volume $\left(\mathrm{mm}^{3}\right)$ was calculated every 7 days for 35 days using the formula: $\mathrm{V}=0.5 \mathrm{x}$ length $\mathrm{x}$ width $^{2}$. After 7 weeks, the mice were euthanized. The tumors were isolated, weighed, photographed.

Immunohistochemical analysis (IHC). Paraffin-embedded tissues were cut into $4 \mu \mathrm{m}$-thick consecutive sections and were then dewaxed in xylene and rehydrated in graded ethanol solutions. Antigen retrieval was performed following the standard procedure. Sections were cooled and immersed in a $0.3 \%$ hydrogen peroxide solution for $15 \mathrm{~min}$ to block endogenous peroxidase activity, and then rinsed in phosphate-buffered saline (PBS) for $5 \mathrm{~min}$. Non-specific labeling was blocked by incubation with $5 \%$ bovine serum albumin at room temperature for $30 \mathrm{~min}$. Sections were then incubated with primary rabbit anti-human antibody against Ki67 (1:200; Santa Cruz Biotechnology, Inc.) at $4^{\circ} \mathrm{C}$ overnight, rinsed with PBS with Tween-20 (PBST), incubated with horseradish peroxidase-conjugated goat anti-rabbit IgG secondary antibody (Santa Cruz Biotechnology, Inc.). The immunohistochemistry results were scored by the percentage of positive detection of the staining.

Statistical analysis. All experiments were repeated 3 times. Unless otherwise indicated, experimental values are expressed as mean \pm standard error mean. Differences between 2 groups were assessed using Student's t-test (two-tailed). Data of $>2$ groups were analyzed using one way analysis of variance with Tukey's post hoc test. Statistical analyses were performed using SPSS 13.0 software (SPSS, Inc., Chicago, IL, USA). $\mathrm{P}<0.05$ was considered to indicate statistical significance.

\section{Results}

Overexpression of HMGN5 was detected in PDAC cell lines and tissues. To investigate the potential role of HMGN5 in PDAC, we first examined the expression level of HMGN5 in the PDAC cell lines Capan-2, PANC-1, SW1990, COLO357 and MIAPaCa-2, as well as in the normal pancreatic epithelial cell line HPDE by qPCR and western blot assay. The results showed that the mRNA and protein expression levels of HMGN5 were significantly higher in PDAC cells compared to HPDE cells (Fig. 1A and B). The highest expression levels of HMGN5 were detected in PANC-1 cells. Furthermore, the expression of HMGN5 was detected in PDAC tissues and matched normal tissues from 15 patients using qPCR. The results showed that the expression level of HMGN5 was higher in PDAC samples than in adjacent normal tissues (Fig. 1C). The increased levels of HMGN5 in PDAC indicate that HMGN5 may serve as oncogene in PDAC.

HMGN5 silencing inhibited PDAC progression in vitro. To examine the role of HMGN5 in human PDAC cell growth, PANC-1 cells were transfected with shcontrol or shHMGN5. Western blot results showed that the expression level of HMGN5 was significantly downregulated in cells transfected with shHMGN5 (Fig. 2A). CCK-8 assay showed that
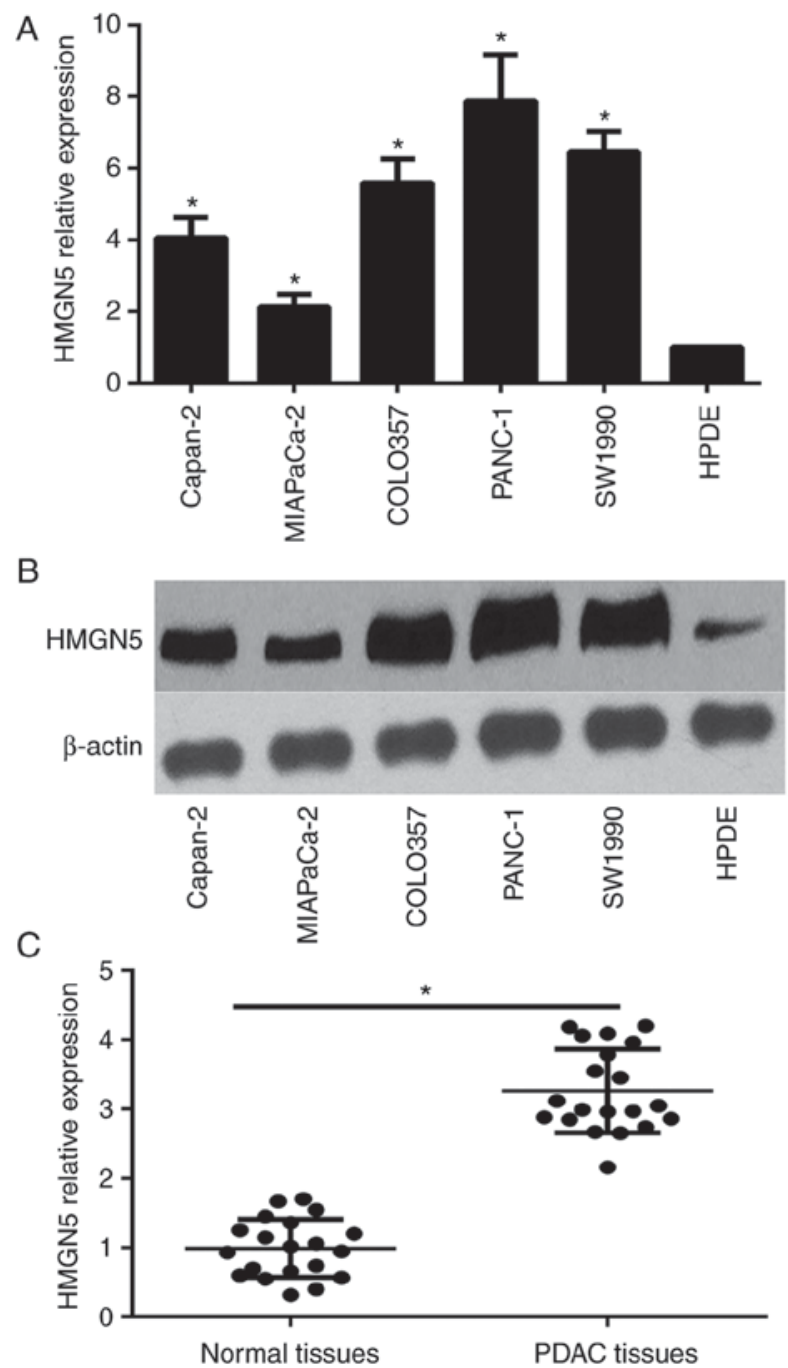

Figure 1. Overexpression of HMGN5 in PDAC cell lines and tissues. (A) RT-qPCR analysis revealed the mRNA expression of HMGN5 in the human PDAC cell lines (PANC-1, Capan-2, SW1990, COLO357 and MIAPaCa-2) and immortalized pancreatic epithelial cell line HPDE. ${ }^{*} \mathrm{P}<0.05$ vs. HPDE. (B) Western blot analysis revealed the HMGN5 protein expression in the human PDAC cell lines (PANC-1, Capan-2, SW1990, COLO357 and MIAPaCa-2) and HPDE. (C) The expression level of HMGN5 in the PDAC tissues and their adjacent normal tissues was detected using RT-qPCR. ${ }^{*} \mathrm{P}<0.05$, as indicated. RT-qPCR, reverse transcription-quantitative polymerase chain reaction; HMGN5, high mobility group nucleosome binding domain 5; PDAC, pancreatic ductal adenocarcinoma.

HMGN5 silencing in PANC-1 cells significantly inhibited cell viability (Fig. 2B). Colony formation assays illustrated that HMGN5 silencing in PANC-1 cells significantly inhibited cell proliferation (Fig. 2C and D).

HMGN5 silencing decreased PDAC metastasis in vitro. Cell metastasis is critical events in the tumor progression. Therefore, the effect of HMGN5 on the migration and invasion of PDAC cell was explored in vitro. PANC-1 cell migration and invasion were investigated through Boyden chamber assay. Results showed that HMGN5 silencing significantly decreased the cell migration and invasion (Fig. 3A-D).

HMGN5 silencing inhibited the tumor growth in vivo. To show the HMGN5 biofunction in promotion of PDAC cell 

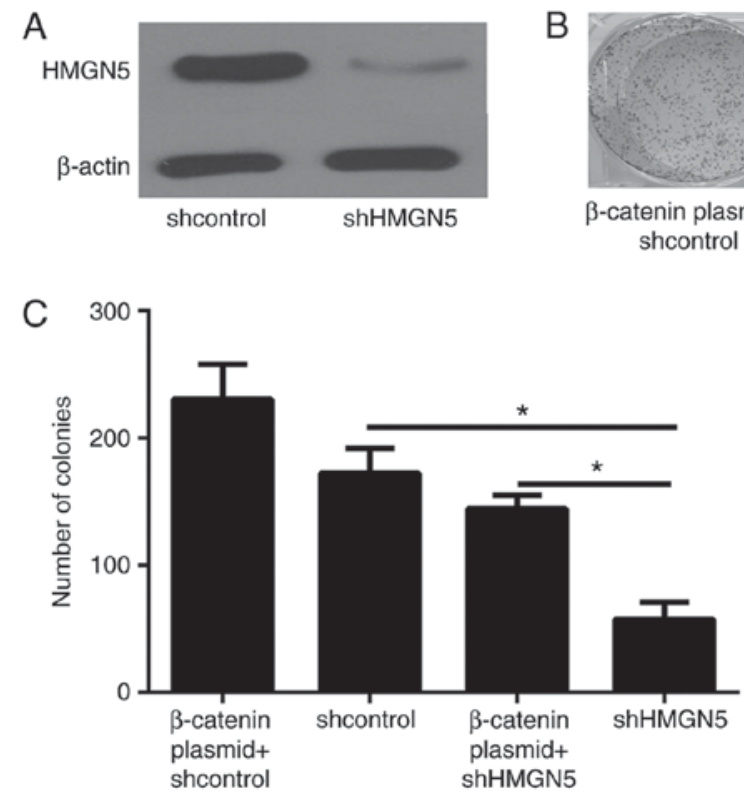

$\mathrm{B}$

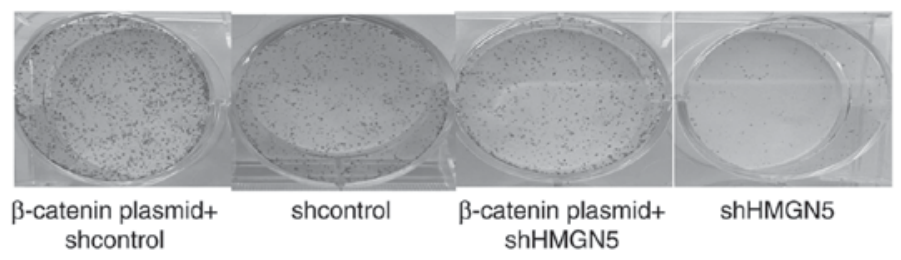

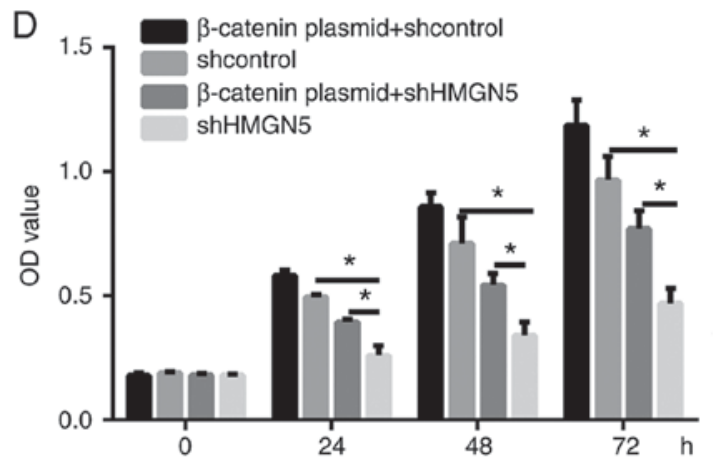

Figure 2. HMGN5 silencing inhibits PDAC progression while $\beta$-catenin overexpression reverses this effect in vitro. PANC-1 cells were transfected with shcontrol or shHMGN5 and $\beta$-catenin overexpression plasmid. (A) The expression levels of HMGN5 and $\beta$-catenin were examined in PDAC cells transfected with shcontrol or shHMGN5 and $\beta$-catenin plasmid by western blotting. (B) The cell proliferation and (C and D) viability were determined in PANC-1 cell via Cell Counting Kit-8 and colony formation assays. "P<0.05, as indicated. HMGN5, high mobility group nucleosome binding domain 5; PDAC, pancreatic ductal adenocarcinoma; sh-, short hairpin RNA; OD, optical density.
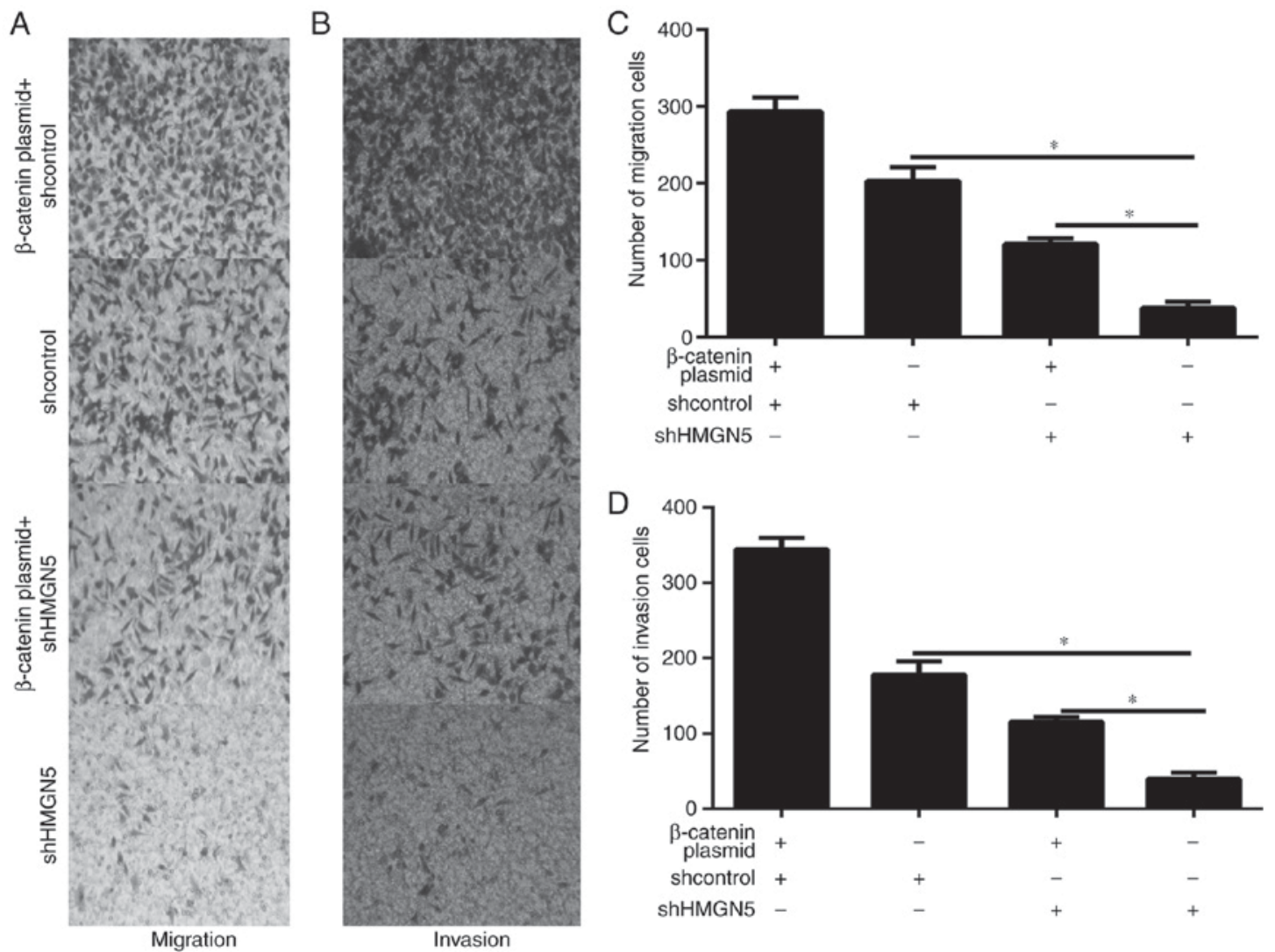

Figure 3. HMGN5 silencing decreases PDAC metastasis while $\beta$-catenin overexpression reverses this effect in vitro. PANC-1 cells were transfected with shcontrol or shHMGN5 and $\beta$-catenin overexpression plasmid. (A and C) Cell migration was determined in PDAC cancer cells via Transwell migration assay. (B and D) Cell invasion was examined in PDAC cancer cells by a Transwell invasion assay (magnification, $\mathrm{x} 200$ ). ${ }^{*} \mathrm{P}<0.05$, as indicated. HMGN5, high mobility group nucleosome binding domain 5; PDAC, Pancreatic ductal adenocarcinoma; sh-, short hairpin RNA.

tumorigenicity in vivo, PANC-1 cells stable expression shcontrol or shHMGN5 was respectively injected into nude mice. The tumor volume was measured every 7 days. At 35 days after cell implantation, the tumors were removed 

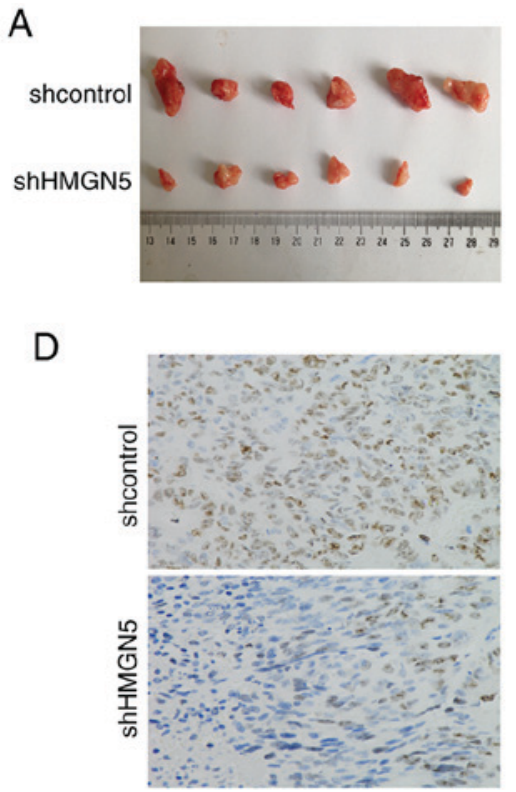

C
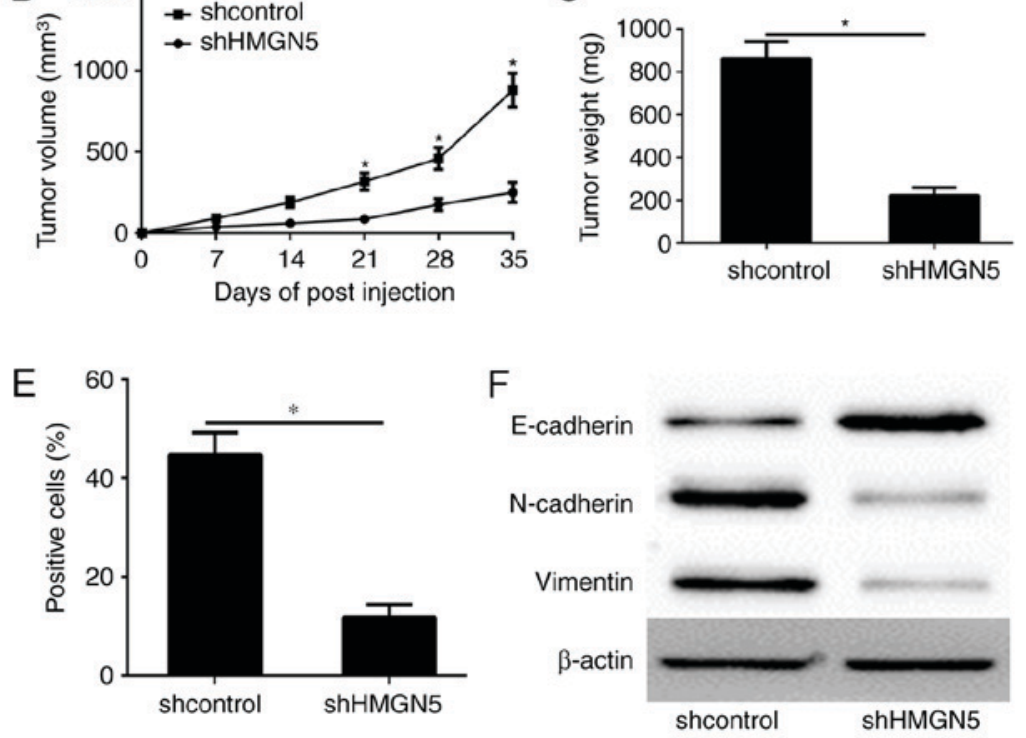

Figure 4. HMGN5 silencing inhibits the tumor growth in vivo. Shcontrol and shHMGN5-transfected PANC-1 cells were injected subcutaneously into athymic nude mice ( $n=6 /$ group). (A) Macroscopic image of tumors excised from mice. (B) Tumor volume of shcontrol and shHMGN5 tumors in mice was measured every 7 days. "P<0.05 vs. shHMGN5 tumors (C) Average weight of resected tumors. (D) Representative images (magnification, $\mathrm{x} 200$ ) of the pancreatic tumor sections stained for Ki67 in shcontrol and shHMGN5 PANC-1 tumors. (E) Quantification of the percentage of Ki67 (+) cells from pancreatic tumor sections. (F) The protein expression of E-cadherin, $\mathrm{N}$-cadherin and vimentin was detected via western blotting. ${ }^{*} \mathrm{P}<0.05$, as indicated. HMGN5, high mobility group nucleosome binding domain 5; sh-, short hairpin RNA.

and photographed (Fig. 4A). Tumor volume and the final weight of excised tumors demonstrated that HMGN5 knockdown had significantly retarded tumor grow th at termination (Fig. 4B and C). Histologic analysis of tumor proliferation showed that HMGN5 silencing had significantly fewer Ki-67 positive cells compared to shcontrol tumors (Fig. 4D and E). These data suggested that HMGN5 may play a critical role in PDAC proliferation and tumor maintenance in vivo.

HMGN5 enhanced EMT in PDAC. EMT is a vital biological process involved in cell differentiation in normal embryonic development and is a potential mechanism for tumour cell metastasis. To address whether HMGN5 has effects on EMT, The expression of the epithelial marker E-cadherin and mesenchymal markers $\mathrm{N}$-cadherin and vimentin was determined in PANC-1 cell transfected with shcontrol and shHMGN5 by western blotting and qPCR. The results showed that the expression of E-cadherin was significantly up-regulated compared with the controls, whereas $\mathrm{N}$-cadherin and vimentin was significantly down-regulated in PANC-1 cell (Fig. 5B-E).

To confirm the above data in vivo, we detected the expression of E-cadherin, $\mathrm{N}$-cadherin and vimentin in the tumour transfected with shcontrol and shHMGN5 by western blotting. The results showed that the expression of E-cadherin was significantly up-regulated in shHMGN5 group, whereas $\mathrm{N}$-cadherin and vimentin was significantly down-regulated in shHMGN5 group (5F).

HMGN5 positively regulated the Wnt/ $\beta$-catenin signaling pathway in PDAC cell. Wnt/ $\beta$-catenin pathway is one of the major signaling pathways involved in EMT and plays an important role in metastasis. Western blot results showed that HMGN5 positively regulated Wnt1 expression in protein (Fig. 5A). Furthermore, we showed the activation of GSK $3 \beta$ and $\beta$-catenin, the canonical pathway of Wnt signaling pathway. The western blot results showed that the amount of p-GSK3 $\beta$ (Fig. 5A) and active $\beta$-catenin (dephosphorylated $\beta$-catenin, Fig. 5A) was dramatically reduced by HMGN5 silencing.

HMGN5 promotes PDAC cell viability, proliferation and metastasis via Wnt/ $\beta$-catenin signaling pathway in vitro. We then investigated whether rescuing HMGN5 silencingmediated-Wnt/ $\beta$-catenin signaling pathway suppression with a $\beta$-catenin overexpression plasmid attenuates the progression inhibition effect of HMGN5 silencing on PDAC cells. The expression of $\beta$-catenin was detected by western blot assay (Fig. 5A). The CCK-8 (Fig. 2B) and colony formation assay (Fig. 2C and D) results showed that restoration of $\beta$-catenin expression reversed the inhibition of cell viability and proliferation by HMGN5 silencing in PANC-1 cell. Furthermore, the Transwell assay showed that $\beta$-catenin overexpression significantly reversed the inhibition of cell migration and invasion by HMGN5 knockdown in PANC-1 cell (Fig. 3A-D). Taken together, these results demonstrated that HMGN5 promoted the PDAC progression via activation of Wnt/ $\beta$-catenin signaling pathway.

Overexpression of $\beta$-catenin counteracted the effect of HMGN5 silencing on EMT in PDAC. We further explored the effect of regulatory relationship of HMGN5 and Wnt/ $\beta$ catenin signaling pathway on EMT of PANC-1 cells. qPCR and western blot assay results showed that the overexpression of $\beta$-catenin counteracted the effect of HMGN5 on EMT as demonstrated by changed expressions of molecular markers associated with EMT (Fig. 5B-E). 

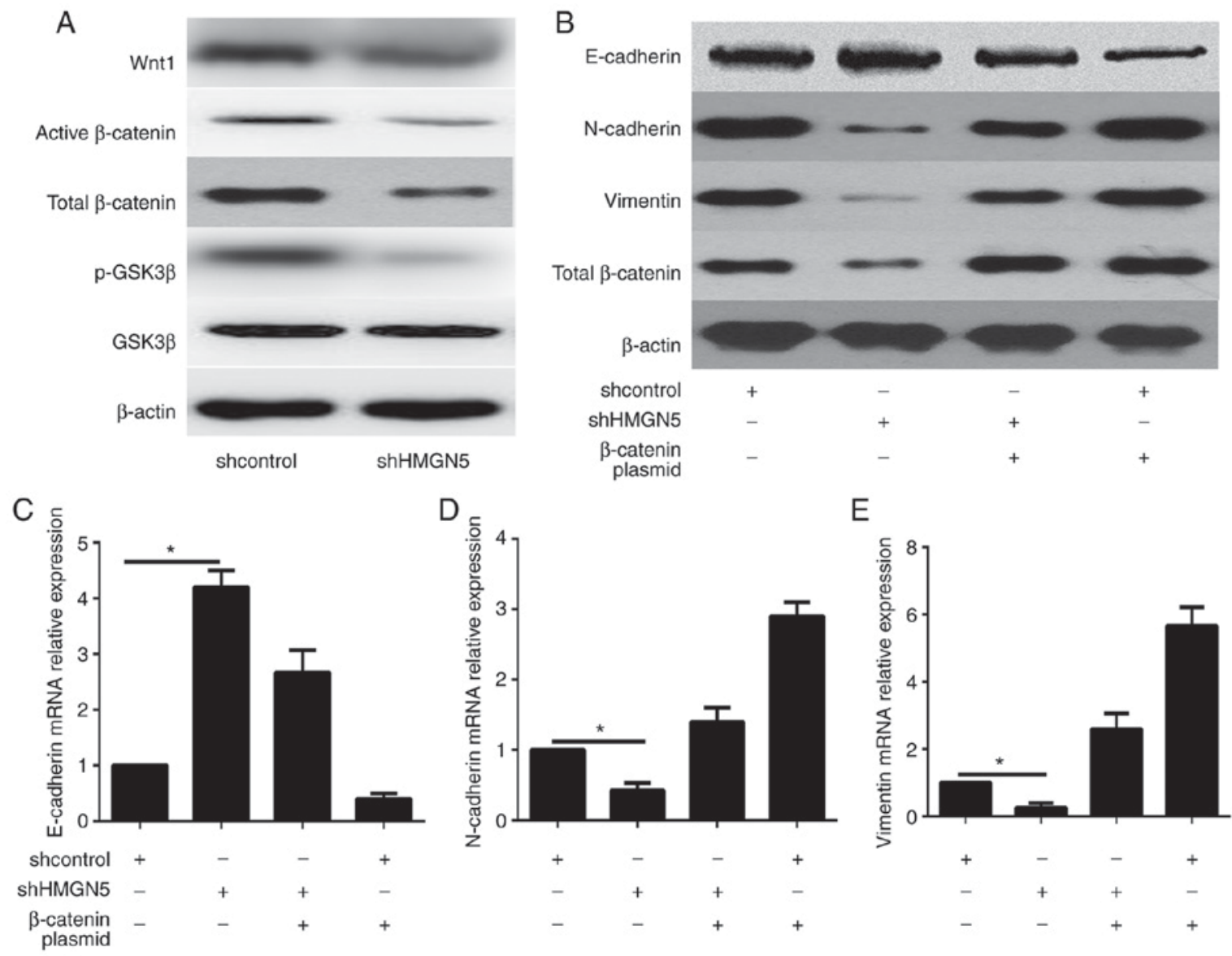

Figure 5. HMGN5 enhances epithelial-mesenchymal transition via the Wnt/ $\beta$-catenin signaling pathway in PDAC cells. PANC-1 cells were transfected with shcontrol or shHMGN5 and $\beta$-catenin overexpression plasmid. (A) Representative western blots of the protein expression levels of Wnt1, total and p-GSK3 $\beta$, and active and total $\beta$-catenin in PANC-1 cells. $\beta$-actin served as the loading control. (B) The protein expression of epithelial and mesenchymal markers was also detected by western blot assay. The mRNA expression of the epithelial and mesenchymal markers, (C) E-cadherin, (D) N-cadherin and (E) vimentin, was detected by reverse transcription-quantitative polymerase chain reaction. ${ }^{*} \mathrm{P}<0.05$, as indicated. HMGN5, high mobility group nucleosome binding domain 5 ; PDAC, pancreatic ductal adenocarcinoma; sh-, short hairpin RNA; p-, phosphorylated; GSK3 $\beta$, glycogen synthase kinase-3 $\beta$.

\section{Discussion}

In the present study, we discovered that the HMGN5 expression was significantly higher in the PDAC cell lines and PDAC tissues than in the normal pancreatic ductal cell line and normal pancreatic tissues. These data suggested that the HMGN5 overexpression may be correlated with PDAC progression.

A previous study has shown that HMGN5 promotes tumour progression in some types of cancer (6-14). However, the role of HMGN5 in pancreatic cancer has not been illustrated. To elucidate the role of HMGN5 in the tumorigenesis of PDAC, we employed the loss of function approach via the knockdown of endogenous HMGN5 expression in the PDAC cells. We firstly showed that HMGN5 silencing decreased the viability of the PANC-1 cells using the CCK- 8 assay. Then, we demonstrated using the colony formation assay that the proliferation rate of the PANC-1 cells significantly decreased. All these data were consistent with those of a previous study that HMGN5 promotes tumour progression.

Metastasis is an important aspect of PDAC. To illustrate the role of HMGN5 in PDAC metastasis, we employed the Transwell assay in vitro. Results showed that the HMGN5 silencing significantly inhibited the migration and invasion of the PANC-1 cells. This result was consistent with that of a previous study. Tumour metastasis is crucially dependent on EMT in pancreatic cancer. EMT is characterized by the mutative expression of an epithelial marker, such as E-cadherin, and mesenchymal markers, such as $\mathrm{N}$-cadherins and vimentin. The EMT process is characterized by the loss of E-cadherin. Such loss creates profound phenotypic alterations that convert the apico-basal polarity of the epithelial cells into front-rear polarity. As a result, the epithelial cells gain mesenchymal characteristics and migration and invasion capacities. Therefore, identifying key molecules involved in the EMT in pancreatic cancer may provide a new therapeutic strategy for treating patients with pancreatic cancer. The correlation of EMT and HMGN5 has not been well illustrated. In the present study, we showed that HMGN5 enhanced the EMT as demonstrated by the downregulation of E-cadherin expression and upregulation of $\mathrm{N}$-cadherins and vimentin in the PANC-1 cells. These data strongly supported that HMGN5 is an activator of EMT in pancreatic cancer.

We illustrated that HMGN5 positively regulated the Wnt signalling pathway. The canonical Wnt signalling pathway is also known as the $\mathrm{Wnt} / \beta$-catenin signalling pathway as $\beta$-catenin is a key transducer of the Wnt signal from the cytoplasm to the nucleus (15). The Wnt/ $/$-catenin signalling 
pathway is an established EMT regulatory signalling pathway due to its maintenance of epithelial integrity and tight adherent junctions (16). Although the Wnt/ $\beta$-catenin signalling pathway is one of the major signalling pathways involved in the EMT in pancreatic cancer (17-19), its regulatory mechanisms still remain obscure. The present study demonstrated for the first time that HMGN5 positively regulated the Wnt/ $\beta$-catenin signalling pathway. In the present study, we firstly demonstrated that HMGN5 promoted the expression of Wnt1, a secreted ligand that activates the Wnt signalling pathways, which are strongly correlated with tumorigenesis and progression. Moreover, we showed that the expression levels of GSK $3 \beta, \beta$-catenin and total $\beta$-catenin were downregulated during HMGN5 silencing. All these data showed that HMGN5 possibly promoted the tumour progression via the activation of the Wnt/ $\beta$-catenin signalling pathway. Furthermore, the reversal of the effect of HMGN5 silencing on tumour growth inhibition and EMT caused by the overexpression of $\beta$-catenin suggested that the effect was through, at least in part, the Wnt/ $\beta$-catenin signalling pathway.

In summary, the present study was the first to demonstrate that the expression of HMGN5 was upregulated in PDAC. We also found that HMGN5 promoted the PDAC cell viability, proliferation, migration, invasion and EMT and suppressed the tumour growth in vivo via the activation of the $\mathrm{Wnt} / \beta$-catenin signalling pathway. These results provided new insights into the mechanism of PDAC progression and suggested that HMGN5 is a potential antitumour agent in the treatment of pancreatic cancer.

\section{Acknowledgements}

Not applicable.

\section{Funding}

No funding was received.

\section{Availability of data and materials}

All data generated or analysed during the present study are included in this published article.

\section{Authors' contributions}

XW and JZ conceived and designed the experiments. JZ and YW conducted all of the experiments, and YW wrote and revised the manuscript. All authors read and approved the final manuscript.

\section{Ethics approval and consent to participate}

The present study was approved by the Medical Ethics Committee of LanLing County Hospital (Shandong, China). All patients included in this research were required to provide written informed consent. The protocols for the animal experiments were approved by the Animal Care and Welfare Committee of LanLing County Hospital and were conducted in strict accordance with the guidelines of the National Animal Welfare Law of China.

\section{Patient consent for publication}

Written informed consent was obtained from each participant.

\section{Competing interests}

The authors declare that they have no competing interests.

\section{References}

1. Siegel RL, Miller KD and Jemal A: Cancer Statistics, 2017. CA Cancer J Clin 67: 7-30, 2017.

2. Melisi D and Budillon A: Pancreatic cancer: Between bench and bedside. Curr Drug Targets 13: 729-730, 2012.

3. Nistico P, Bissell MJ and Radisky DC: Epithelial-mesenchymal transition: General principles and pathological relevance with special emphasis on the role of matrix metalloproteinases. Cold Spring Harb Perspect Biol 4: pii: a011908, 2012.

4. Gaianigo N, Melisi D and Carbone C: EMT and treatment resistance in pancreatic cancer. Cancers 9: pii: E122, 2017.

5. Rochman M, Malicet $C$ and Bustin M: HMGN5/NSBP1: A new member of the HMGN protein family that affects chromatin structure and function. Biochim Biophys Acta 1799: 86-92, 2010.

6. Ji SQ, Yao L, Zhang XY, Li XS and Zhou LQ: Knockdown of the nucleosome binding protein 1 inhibits the growth and invasion of clear cell renal cell carcinoma cells in vitro and in vivo. J Exp Clin Cancer Res 31: 22, 2012.

7. Chen P, Wang XL, Ma ZS, Xu Z, Jia B, Ren J, Hu YX, Zhang QH, Ma TG, Yan BD, et al: Knockdown of HMGN5 expression by RNA interference induces cell cycle arrest in human lung cancer cells. Asian Pac J Cancer Prev 13: 3223-3228, 2012.

8. Su B, Shi B, Tang Y, Guo Z, Yu X, He X, Li X, Gao X and Zhou L: HMGN5 knockdown sensitizes prostate cancer cells to ionizing radiation. Prostate 75: 33-44, 2015.

9. Jiang W, Zheng J, Yu T and Wang J: Overexpression of microRNA-495 suppresses the proliferation and invasion and induces the apoptosis of osteosarcoma cells by targeting highmobility group nucleosome-binding domain 5. Oncol Rep 38: 1099-1107, 2017

10. Cao Y, Zhang L, Wei M, Jiang X and Jia D: MicroRNA-409-3p represses glioma cell invasion and proliferation by targeting high-mobility group nucleosome-binding domain 5. Oncol Res 25: 1097-1107, 2017.

11. Weng M, Song F, Chen J, Wu J, Qin J, Jin T and Xu J: The highmobility group nucleosome-binding domain 5 is highly expressed in breast cancer and promotes the proliferation and invasion of breast cancer cells. Tumour Biol 36: 959-966, 2015.

12. Gan Y, He L, Yao K, Tan J, Zeng Q, Dai Y, Liu J and Tang Y: Knockdown of HMGN5 increases the chemosensitivity of human urothelial bladder cancer cells to cisplatin by targeting PI3K/Akt signaling. Oncol Lett 14: 6463-6470, 2017.

13. Liu X, Ma W, Yan Y and Wu S: Silencing HMGN5 suppresses cell growth and promotes chemosensitivity in esophageal squamous cell carcinoma. J Biochem Mol Toxicol 31, 2017.

14. Shi Z, Tang R, Wu D and Sun X: Research advances in HMGN5 and cancer. Tumour Biol 37: 1531-1539, 2016.

15. Nusse $\mathrm{R}$ and Clevers $\mathrm{H}$ : Wnt/ $\beta$-catenin signaling, disease, and emerging therapeutic modalities. Cell 169: 985-999, 2017.

16. Huang L, Wu RL and Xu AM: Epithelial-mesenchymal transition in gastric cancer. Am J Transl Res 7: 2141-2158, 2015.

17. Peng L, Liu Z, Xiao J, Tu Y, Wan Z, Xiong H, Li Y and Xiao W: MicroRNA-148a suppresses epithelial-mesenchymal transition and invasion of pancreatic cancer cells by targeting Wnt10b and inhibiting the Wnt/ $/$-catenin signaling pathway. Oncol Rep 38: 301-308, 2017.

18. Bao Z, Xu X, Liu Y, Chao H, Lin C, Li Z, You Y, Liu N and Ji J: CBX7 negatively regulates migration and invasion in glioma via Wnt/ $\beta$-catenin pathway inactivation. Oncotarget 8: 39048-39063, 2017.

19. Nakamoto M and Hisaoka M: Clinicopathological Implications of Wingless/intl (WNT) signaling pathway in pancreatic ductal adenocarcinoma. J UOEH 38: 1-8, 2016. 